\title{
Diagnóstico de muerte encefálica en la legislación peruana actual
}

\author{
Luis Deza Bringas
}

La ley 28189, llamada Ley General de Donación y Trasplante de Órganos y/o Tejidos Humanos, expedida el 16 de marzo de $2004,{ }^{1}$ y su reglamento contenido en el decreto supremo 014-2005-SA, publicado en mayo de 2005, ${ }^{2}$ son los nuevos instrumentos legales que regulan en nuestro país las actividades y procedimientos relacionados con la obtención y utilización de órganos y/o tejidos humanos para fines de donación y trasplante y su seguimiento. El proyecto del citado reglamento fue elaborado por una comisión integrada por representantes de los ministerios de Salud, Educación, Defensa e Interior, así como por representantes del Seguro Social de Salud, del Colegio Médico del Perú y de la Asociación de Clínicas Privadas, y luego presentado para su aprobación por el Poder Ejecutivo.

Como médico neurólogo interesado en el tema, he considerado pertinente analizar específicamente el título II de dicho reglamento, denominado el «Diagnóstico de muerte», y opinar acerca de él, señalando sus virtudes y errores: analizaré concretamente los artículos que van del 3 al 10 de dicho título. Mi finalidad es la de contribuir a optimizar la normatividad jurídica nacional sobre asuntos de tanta significación, como son la determinación de la muerte y los criterios que deben usarse para certificar su diagnóstico, ya sea mediante la verificación del cese irreversible de la función cardiorrespiratoria — cuyo protocolo diagnóstico ha sido ignorado en la reciente ley—o la desaparición de la actividad encefálica, denominada muerte encefálica en el mencionado título II.

\footnotetext{
Servicios de Salud de la Pontificia Universidad Católica del Perú y Academia Nacional de Medicina. Correo: Ideza@pucp.edu.pe

1 Diario oficial El Peruano (18-3-2004): ley 28189. Ley General de Donación y Trasplante de Órganos y/o Tejidos Humanos.

2 Diario oficial El Peruano (27-5-2005): Reglamento de la Ley General (28189) de Donación y Trasplante de Órganos y/o Tejidos Humanos.
} 
El tercer artículo del reglamento inicia la más reciente disposición promulgada en el Perú, con el fin de reducir los precedentes vacíos de nuestra legislación acerca del tema en discusión y, asimismo, optimizar su contenido.

Artículo $3^{\circ}$. Diagnóstico de muerte

Se considera muerte para efectos del presente Reglamento al cese irreversible de la función encefálica o la función cardiorrespiratoria, de acuerdo con los protocolos que establecen en el presente Reglamento. El diagnóstico de la muerte de una persona es de responsabilidad del médico que la certifica.

Años antes, el asunto fue atendido oficialmente en junio de 1982, al promulgarse la ley 23415 sobre los «[...] transplantes de órganos y tejidos de cadáveres así como los transplantes de órganos y tejidos de personas vivas». ${ }^{3} \mathrm{El}$ artículo 5 de la misma calificó como muerte — «[...] para los fines de la presente Ley" — tanto el cese definitivo e irreversible de la actividad cerebral —no se utilizó la entonces común denominación de muerte cerebral— como la ausencia, sin retorno, de la función cardiorrespiratoria. Se destacó de esta manera que la muerte o término de la vida de una persona, aunque es un hecho singular, admite sin embargo que para determinar su ocurrencia, sean aceptables cualquiera de los dos diagnósticos clínicos citados.

Pero en la ley 23415 no se incluyeron los recomendables protocolos o criterios específicos que debían cumplirse y exigirse legalmente para diagnosticar la muerte de una persona, mediante la verificación del cese irreversible de la función cardiorrespiratoria o de la función cerebral, confiando únicamente la responsabilidad de la constatación al médico que certificaba el hecho. De otro lado, al haberse incluido, expresamente, la aceptación jurídica de la muerte (cerebral) entre las normas aplicables a los transplantes de órganos, se llegó a la equívoca situación en la cual una persona declarada en muerte cerebral en el Perú no estaba legalmente muerta para todos sus efectos, sino únicamente para los fines de la ley de trasplantes. Luego, el Código Civil peruano promulgado en $1984^{4}$ trató el tema muy escuetamente, señalando llanamente que la muerte pone fin a la persona, sin extenderse en consideraciones acerca de la definición de la muerte y los criterios para su diagnóstico, remarcando únicamente que ella debía ser certificada por un médico.

Por tal motivo, en 1985 publicamos un extenso artículo que abarcó diversos aspectos médicos y legales de la muerte cerebral en el Perú. ${ }^{5}$ Este documento se

3 Diario oficial El Peruano (4-6-82): ley 23415. Los transplantes de órganos y tejidos de cadáveres así como los transplantes de órganos y tejidos de personas vivas estarán regidos por la presente ley.

4 Código Civil peruano. Libro I. Derecho de las personas. Sección primera. Artículo 5. Derecho a la vida. 1984

5 Deza Luis y E. Ala LunA. «Aspecto médicos y legales de la muerte cerebral». Acta Médica Peruana, 1985, 12, pp. 68-78. (Este mismo artículo fue reproducido con autorización en la revista del foro). 
enriqueció con el abundante material bibliográfico internacional acerca del tema, obtenido, mayormente, mediante el valioso apoyo de la biblioteca de la Universidad de Texas. Su idea central fue la de reclamar la necesidad de incorporar en la legislación peruana las normas que fijaran claramente la identificación de la muerte cerebral con la muerte de la persona. A partir de tal reconocimiento, recién podrían hacerse efectivas las innumerables consecuencias jurídicas que ocasiona la ausencia de vida, siendo solo una de ellas, en los casos aplicables, las relacionadas a los trasplantes de órganos cadavéricos. De otro lado, solicitamos el perfeccionamiento de la ley de trasplantes, mediante la inclusión en ella del faltante protocolo que debía exigirse para aceptar el diagnóstico de muerte cerebral, el cual además debía tener características diferentes en adultos y en niños.

Cabe recordar que ni el Código Civil ni la ley 23415 reconocieron, taxativamente, a la muerte cerebral como muerte de la persona, sino solo en lo concerniente a las necesidades de los transplantes de órganos y tejidos en humanos. Pero además, en la redacción de los citados documentos no se señalaron los criterios que debían cumplirse para validar la certificación de la muerte cerebral. En oposición, esta importante norma era infaltable en esos años en todos los países en los cuales se reconocía legalmente a la muerte cerebral como muerte de la persona, ya que el estricto acatamiento de tales criterios fortalecía la requerida profesionalización del diagnóstico médico, mermaba las ocasionales suspicacias éticas que podían suscitarse en la materia, y por cierto reducía no pocas e injustificadas denuncias judiciales.

El proceso penal seguido en contra del cardiocirujano Bellizi, quien en 1968 realizó el primer transplante cardíaco en Buenos Aires, fue un ejemplo patético de los peligros de la insuficiente normatividad jurídica en la Argentina de esa época. En efecto, cuando a los pocos días del transplante falleció el paciente receptor del órgano, el doctor Bellizi fue doblemente acusado de homicidio simple del donante, por haber actuado dolosamente con la intención de extirpar el corazón del paciente, y de homicidio culposo del receptor, por atribuirse la muerte del mismo a la impericia del cirujano que realizó el procedimiento. La posición facultativa, en apoyo del doctor Bellizi, fue que en el momento del transplante, el sujeto donante se encontraba clínicamente muerto, en el estado irremediable e irreversible, denominado médicamente muerte cerebral. La ruidosa controversia generada en torno al asunto solo se atenuó cuando, poco después, se estableció en Argentina el reconocimiento legal de la muerte cerebral como muerte de la persona.

Históricamente, a partir de $1959^{6}$ se dieron a conocer en la bibliografía médica mundial algunos reportes aislados acerca de pacientes que se encontra-

Mollaret P. y M. Goulon. «Le coma dépassé». En Revue Neurologique 101, 1959, pp. 3-15; Wertheimer P.; Jouvet, M.; Descotes J. «A propos du diagnostic de la mort du systéme nerveux 
ban en la situación que posteriormente fue llamada muerte cerebral, esencialmente debido al planteamiento conceptual dado a conocer en 1966 por la Academia Francesa de Medicina. De acuerdo al mismo, la muerte de una persona podía ser declarada como tal, si el sujeto afectado tenía parálisis irreversible de la función cerebral, y esta a su vez era comprobada mediante un cuidadoso examen clínico, sumado a la ausencia de actividad eléctrica cortical espontánea, registrada por un electroencefalógrafo. ${ }^{7}$

Recordemos que desde la Antigüedad, el juicio estándar o tradicional utilizado mayormente para verificar la ausencia de vida o la muerte de un ser, era la constatación del cese irreversible de la función cardiorrespiratoria y algunos otros signos cadavéricos, descritos detalladamente en los libros de Medicina Legal, como son el enfriamiento corporal, las livideces cadavéricas, la rigidez, la putrefacción, etcétera. Pero cuando se introdujo en el cuidado de los enfermos graves, el uso de los ventiladores o respiradores mecánicos sumados a una serie extensa de recursos técnicos, disponibles en las unidades hospitalarias de terapia intensiva, se hizo cada vez más frecuente encontrar pacientes afectados primariamente por muy severas lesiones cerebrales, de carácter irremediable, quienes no obstante estar muertos por carecer completamente de función cerebral y sin ninguna posibilidad de recuperación, seguían de modo ficticio respirando artificialmente por efecto de los aparatos de ventilación mecánica. Además, desde que el corazón, por su actividad autónoma y el apoyo médico farmacológico puede mantenerse funcionando por algún tiempo, impulsando la circulación de la sangre por todo el cuerpo, se completaba la mencionada ilusoria apariencia de vida del cadáver en el estado que fue denominado muerte cerebral. Tal situación fue descrita dramáticamente por Christopher Pallis, un distinguido conocedor del tema en los siguientes términos: «Un cerebro muerto en un cuerpo cuyo corazón está todavía latiendo es uno de los productos más macabros de la tecnología moderna». ${ }^{8}$

En sentido estricto, la referida opinión de la Academia Francesa de Medicina sintetizó el pensamiento que desde varios años atrás sostenían los médicos, remarcando que la muerte cerebral significaba la muerte clínica de una persona. La consecuencia inmediata fue el pedido casi generalizado de que en todos los países la muerte cerebral fuera reconocida como muerte de la persona, no solo desde el punto vista médico sino también legal. Adicionalmente, el concepto de muerte cerebral adquirió indiscutible credibilidad cuando varias prestigiosas instituciones médicas propusieron los criterios, racionalmente aceptables,

dans le comas avec arréet respiratoire traités par respiration artificielle». En Press Med 67, 1959, pp. 87-88.

7 Seidler, Eduard. «Progreso y límites de la medicina actual». En Crónica de la Medicina. España: Editorial de Plaza \& Janés, 1993, p. 531.

8 PALLIS, C. «ABC of brain stem. Reappraising death». En Br Med J 1982, 285, pp. 1409-1412. 
para llegar al diagnóstico de tal condición. Así, en 1968 se publicó el parecer de la Universidad de Harvard sobre el tema, ${ }^{9}$ en 1971 el de la Universidad de Minnesota, ${ }^{10}$ en 1976 el del Medical Royal College y sus facultades en el Reino Unido, ${ }^{11}$ y en 1981 se conoció la propuesta de la Comisión Presidencial de los Estados Unidos. ${ }^{12}$ Por consiguiente, cuando en 1985 publicamos el trabajo ya citado, ${ }^{13}$ resaltamos el hecho de que en los países en los cuales se reconocía legalmente a la muerte cerebral como el término de la vida, se habían fijado, casi sin excepción, los criterios o protocolos para validar su diagnóstico. Aunque se notaban algunas diferencias entre ellos, estas eran solo menores, prevaleciendo el consenso alrededor de seis pautas básicas que se muestran en la tabla, las cuales siguen aceptándose hasta ahora, aunque con varias y explicables actualizaciones que comentaremos más adelante.

\section{Criterios generales para determinar la muerte cerebral}

1. Paciente sin perceptividad ni reactividad consciente, como consecuencia de haber sufrido una lesión estructural grave e irremediable.

2. Ausencia de respiración espontánea — necesitando el uso continuo de un respirador-.

3. Ausencia de reflejos del tronco encefálico y de cualquier otro reflejo no espinal.

4. Exclusión de situaciones de muerte cerebral aparente.

5. Reevaluaciones.

6. Silencio electrocerebral, demostrable por el electroencefalograma — prueba no indispensable - y realización de alguna otra prueba confirmatoria en los casos de duda acerca del diagnóstico de muerte cerebral.

La mencionada ley 23415 de 1982 siguió vigente en el Perú hasta junio de 1987, en que la ley 24703 modificó varios de sus artículos. Esta nueva norma

9 Definition of irreversible coma: report of the Ad Hoc Committee of Harvard Medical School to examine the definition of brain death. JAMA 1968; 205: 337-340.

10 Mohandas A, Chou SN. Brain death. A clinical and pathological study. J Neurosurg 1971; 35: 211-218.

11 Diagnosis of brain death: statement issued by the honorary secretary of the Conference of Medical Royal Colleges and their Faculties in the United Kingdom. BMJ 1976; 2: 11871188.

12 President's Comrnission for the study of Ethical Problems in Medicine and Biomedical and behavioral Research. Defining death: a report on the medical, legal and ethical issues in the determination of death. Washington DC: Government Printing Office, 1981.

13 Deza Luis, Ala Luna E. Aspecto médicos y legales de la muerte cerebral. Acta médica peruana, 1985; 12: 68-78. (Este mismo artículo con, autorización, fue reproducido en la Revista del Foro). 
fue completada en mayo de 1988 al publicarse el texto del Reglamento de la Ley de Transplantes 23415 y su modificatoria ley $24703 .{ }^{14} \mathrm{El}$ capítulo III contiene, detallado, el protocolo exigido para validar el diagnóstico de muerte cerebral en nuestro país, el cual debía ser siempre certificado con la firma de tres médicos, quienes debían realizar dos evaluaciones consecutivas, la segunda no antes de las seis horas. Tal disposición, de cumplimiento obligatorio, cubrió el reclamado vacío en la normatividad jurídica peruana sobre los criterios de diagnóstico que debían cumplirse para verificar la muerte cerebral. Pero por otro lado, al seguir incluyendo a la muerte cerebral en la ley de transplantes, se mantuvo la falta de claridad precedente con respecto de las personas declaradas en muerte cerebral, pero cuyos órganos no estaban disponibles para transplantes, las cuales podrían ser entonces consideradas legalmente vivas (¿?). Pero además, el reglamento de la ley 23415 tuvo varios deplorables errores conceptuales y de redacción, mayormente notorios al fundamentarse el tópico de los criterios para constatar la muerte cerebral. A ellos aludimos en un detallado artículo, publicado poco tiempo después de la promulgación del precepto legal citado, ${ }^{15}$ siendo ahora innecesario recordarlos.

Finalmente, en julio de 1997 se promulgó la Ley General de Salud, ley 26842. ${ }^{16}$ Este importante documento derogó varias disposiciones anteriores sobre la salud en nuestro país, entre ellas el antiguo Código Sanitario 17505, aportando de otro lado precisas y oportunas normas sobre los deberes, derechos y responsabilidades concernientes a la salud individual y colectiva. El título tercero "Del fin de la vida», en su artículo 108 dice lo siguiente:

La muerte pone fin a la persona. Se considera ausencia de vida al cese definitivo de la actividad cerebral, independientemente de que algunos de sus órganos o tejidos mantengan actividad biológica y puedan ser usados con fines de transplante, injerto o cultivo. El diagnóstico fundado de cese definitivo de la actividad cerebral verifica la muerte. Cuando no es posible establecer tal diagnóstico, la constatación de paro cardiorrespiratorio irreversible confirma la muerte.

A partir de este conciso precepto, la muerte cerebral fue por fin admitida en el Perú como muerte legal de la persona, para todos sus efectos y no solo para amparar judicialmente a los transplantes de órganos cadavéricos. Es pertinente

14 Diario oficial El Peruano (31-5-1988): Reglamento de la ley 23415, modificada por la ley $N^{\circ} 24703$.

15 Deza Luis, Salinas C. «La muerte cerebral y sus implicancias jurídicas en el Perú». En Revista del Instituto Peruano de Seguridad Social 1991; 1: 45-54.

16 Diario oficial El Peruano (20-7-1997): Ley General de Salud № 26842. Título III Del fin de la vida. 
referir que cuando una persona es catalogada en el estado de muerte cerebral, o usando otros términos, si una persona es declarada muerta por cumplir con los criterios exigidos para tal diagnóstico, el médico que certifica el hecho debe, por motivos obvios, disponer lo conveniente para evitar el mantenimiento innecesario de las, además, costosas medidas terapéuticas hospitalarias que estaban en uso hasta ese momento, incluyendo la conexión al equipo de ventilación mecánica. Luego, al expedirse el indispensable certificado de defunción, el cadáver es usualmente encaminado hacia una de dos opciones: la primera, que es la más frecuente, termina rápidamente con la entrega del fallecido a sus familiares, para el velorio y la inhumación correspondiente; y la segunda, ciñéndose desde luego de modo irrestricto al mandato de la correspondiente ley, puede convertir a alguno de tales cadáveres en donantes de órganos o tejidos antes de su entierro.

Es pues evidente que la determinación de la muerte cerebral es un diagnóstico médico cuya validación es regulada legalmente y que la donación y el trasplante de órganos son una opción que puede o no darse de acuerdo a ciertas circunstancias, asimismo ordenadas por ley. Otro aspecto encomiable del artículo 108 de la Ley General de Salud es la precisión en su redacción, cuando dice que la muerte pone fin a la persona y que el cese irreversible de la actividad cerebral se considera ausencia de vida. Esta idea es exacta, ya que en último término la muerte de la persona llega cuando el cerebro deja de funcionar totalmente y de manera irremediable.

Las patologías de naturaleza mortal que afectan al hombre son múltiples. No obstante, pueden separarse en dos grupos: el primero se caracteriza porque las diversas lesiones causantes dañan directamente el cerebro provocando la llamada muerte cerebral o muerte encefálica. Esta se caracteriza por el compromiso global de los hemisferios cerebrales, las zonas vecinas subcorticales, el cerebelo y el tronco encefálico; por tal motivo hay pérdida de las funciones del sistema nervioso, incluidas las relacionadas con el control de la respiración, la circulación de la sangre y la temperatura corporal. Es entonces comprensible que al producirse la muerte encefálica, salvo circunstancias excepcionales o por mediación de los métodos de cuidados médicos intensivos ya mencionados, el proceso deletéreo avance progresivamente en muy corto tiempo hasta comprometer al resto de órganos. De otro modo, en el segundo grupo las igualmente incurables patologías causan indirectamente la muerte del cerebro y desde luego de la persona, debido a que deprimen o suprimen el aporte de oxígeno y nutrientes, que en cantidad y calidad crítica por unidad de tiempo necesita continuamente el tejido cerebral para funcionar. Si el trastorno no es revertido oportunamente, en cuestión de minutos empieza la irremediable necrosis de la masa encefálica y luego en secuencia temporal, el proceso deletéreo avanza progresivamente al resto de tejidos y órganos del cuerpo, cuyo tiempo 
de resistencia a la ausencia de sangre y nutrientes esenciales difiere en cada uno de ellos, aunque invariablemente es mayor que el del cerebro.

Sobre la base de la observación de este inexorable curso, precediendo a la putrefacción cadavérica, ya en antiguos tratados de Medicina Legal se insistía en recordar que la muerte de un ser humano no es un evento sino un proceso, aludiendo a la diferencia temporal que caracteriza a la necrosis final de los diferentes órganos y tejidos del cuerpo, ocupando las uñas y el cabello el último lugar. Por consiguiente, aunque la muerte o ausencia de vida de una persona puede ser consecutiva a múltiples patologías, ella ocurre en último término solo cuando se produce el cese definitivo de la actividad cerebral. Recordemos que la comprobación de tal estado se realiza mediante alguno de los dos criterios de diagnóstico ya mencionados, los cuales, debido a su importancia, serán explicados con un par de ejemplos a continuación, reiterando que se trata estrictamente de la manera dual permitida para constatar la ausencia de vida.

En el primer ejemplo, una persona tiene súbitamente una grave hemorragia cerebral de tipo subaracnoidea, por ruptura espontánea de un aneurisma. Como consecuencia, su cerebro es dañado directamente por el sangrado masivo dentro del cráneo. Con el fin de evitar su agravamiento, el enfermo es conectado a un aparato de respiración artificial y se le brindan además otras sofisticadas atenciones, pero no obstante, la lesión cerebral causada por la hemorragia es irremediable e irreversible, llegando el paciente, desafortunadamente, a la condición, médicamente calificada, como muerte cerebral. La verificación del fin de la vida en esta situación está sujeta a las exigencias clínicas y legales plasmadas en protocolos o criterios uniformes requeridos en cada país para el diagnóstico de muerte cerebral. Las características nacionales de ellos difieren algo, aunque existe en su redacción una base de consenso internacional, algo similar al contenido del recuadro sobre los criterios para determinar la muerte cerebral. El cumplimiento de los protocolos de diagnóstico asegura la idoneidad del acto, y el consenso de su contenido en cada país evita las opiniones personales que pueden llevar a posiciones ridículas, como que una misma persona declarada fallecida, por estar en muerte cerebral, siguiendo el protocolo particular de algún hospital, simultáneamente no reúna los requisitos para tal diagnóstico, es decir se le considere viva de acuerdo a los criterios propios de otro hospital de su misma patria. Más adelante volveremos a esta materia, ya que los protocolos de muerte cerebral de las diferentes naciones son algo disímiles en su contenido, y asimismo, a través del tiempo ellos han tenido en muchos países, incluyendo el nuestro, necesarias modificaciones.

El segundo ejemplo es el de una persona que tiene hipertensión arterial crónica y súbitamente le sobreviene un infarto cardíaco en su hogar. Es trasladada lo más rápidamente a un hospital, pero desdichadamente a su arribo, 
cuarenta minutos después del inicio del problema, el médico de guardia en emergencia ya no puede hacer otra cosa que constatar el fallecimiento. Para el efecto, verifica básicamente la ausencia de latidos cardíacos y de respiración. En este ejemplo, el criterio para el diagnóstico del fin de la vida es el cese definitivo e irreversible de la función cardiorrespiratoria. Este juicio diagnóstico, diferente al de la muerte cerebral, es ocasionalmente llamado tradicional, ya que ha sido usado por los médicos desde tiempos remotos, junto a los citados signos cadavéricos. Aquí, es forzoso destacar que la persona del ejemplo no murió cuando se le paralizó el corazón o cuando dejó de respirar, sino cuando a consecuencia de tal cese, murió el cerebro indirectamente por no haber recibido el aporte sanguíneo crítico imprescindible para su supervivencia.

Es evidente, pues, que en la legislación peruana está bien precisada la definición de muerte de la persona o el fin de la vida, estando además puntualizado que ella puede ser determinada tanto por el diagnóstico de muerte cerebral como por el cese de la función cardiorrespiratoria. No obstante, en nuestra normatividad hay todavía un vacío jurídico parcial en lo concerniente a los protocolos o criterios que deben cumplirse para validar, separadamente, cualquiera de las dos posibilidades. De hecho, en la novísima ley 28189 y su reglamento expedido en mayo del 2005 se especifica en el artículo $3^{\circ}$ que $"[\ldots]$ se considera muerte para efectos del presente Reglamento al cese irreversible de la función encefálica o la función cardiorrespiratoria, de acuerdo con los protocolos que se establecen en el presente Reglamento». Pero en realidad, en el documento no consta el protocolo para verificar la ocurrencia de la muerte cardiorrespiratoria, sino únicamente el recomendado para certificar la muerte encefálica. Por consiguiente, los comentarios que siguen están referidos a esta solitaria obligación.

Antes de iniciar el análisis del protocolo, considero pertinente destacar que los autores del reglamento han preferido usar el término muerte encefálica en reemplazo de la anterior y muy difundida denominación de muerte cerebral, seguramente atendiendo a las consideraciones semánticas sustentadas en años recientes por algunos autores extranjeros. ${ }^{17}$ Pero no puede aceptarse que habiendo adoptado en el reglamento de nuestra ley el nombre de muerte encefálica, de modo incomprensible, al final del mismo, en el anexo 1 , se incluya como modelo impreso, de uso obligatorio, el discordante encabezamiento: «Acta de muerte cerebral».

\section{Artículo $4^{\circ}$. Muerte encefálica}

Se considera muerte encefálica al cese irreversible de las funciones del tronco encefálico cuyo protocolo de diagnóstico se establece en los artículos $7^{\circ}$ y $8^{\circ}$ del presente Reglamento. El acta de comprobación de muerte encefálica es

17 Machado C. ¿Defendemos una visión encefálica de la muerte?. Rev Neurol 2002; 35: 387-396. 
responsabilidad del Director del Establecimiento o su representante, el Neurólogo o Neurocirujano y el Médico tratante.

Este artículo mantiene las encomiables características de la normatividad precedente al respecto. Es obvio que el diagnóstico de muerte encefálica tiene mayor seguridad, cuando en todas partes se exige la opinión concordante de tres médicos para su validación, quienes deben estar presentes en el momento del examen y luego firmar, si fuera conforme, el acta de comprobación de muerte encefálica según el artículo 4, o en sentido literal estricto, el acta de muerte cerebral documentada en nuestro discrepante anexo 1 ya mencionado.

Articulo $5^{\circ}$.- Certificación de la muerte encefálica

La Certificación de la Muerte Encefálica, previa a los procedimientos destinados a la utilización de órganos o componentes anatómicos con fines de trasplante, será indispensable sólo en caso de trasplante de riñón, corazón, hígado, páncreas, intestino y pulmones. En caso de tejidos como piel, córnea, huesos tendones y articulaciones será suficiente la certificación usual de muerte, por parte del médico.

La redacción de este artículo separa con nitidez la certificación de la muerte encefálica — validada por tres médicos y concordante con el protocolo específico-, exigida en las donaciones de órganos cadavéricos, de la certificación usual o diagnóstico tradicional de muerte, basado en la ausencia irreversible de la función cardiorrespiratoria, cuya certificación no está obligada al cumplimiento de protocolo legal alguno: basta para su aceptación diagnóstica la responsable verificación de un solo médico. Esta segunda modalidad de certificación de muerte ha sido redactada, posiblemente, pensando que su aplicación es y será solo para los casos de trasplante de tejidos, con exclusión de los trasplantes de órganos cadavéricos. Sin embargo, algunas publicaciones recientes aportan evidencias que contradicen tal idea.

De hecho, hay varios reportes comunicando que debido al desarrollo de nuevas técnicas para preservar órganos y tejidos, es factible realizar también trasplantes de órganos provenientes de cadáveres cuyo diagnóstico causal es el cese de la función cardiorrespiratoria. ${ }^{18}$ Por esta razón, en la legislación sobre donación de órganos y tejidos de algunos países extranjeros se incluyen dos convenientes protocolos: uno para el cese primario de la función encefálica y otro para el cese tradicional cardiorrespiratorio. ${ }^{19} \mathrm{Al}$ parecer, los autores del

Molina-Martínez F J, Tarongí-Sánchez S, Barceló-Artigues M I, Barceló-Roselló A. Muerte encefálica y donación de órganos. Revisión a la luz de la legislación española actual. Rev Neurol 2003; 36: 771-780.

19 Real Decreto 2070/1999, del Ministerio de la Presidencia, por el que se regulan las actividades de obtención y utilización clínica de órganos humanos y la coordinación territorial en materia de donación y trasplante de órganos y tejidos. BOE número 3, enero del 2000; 179-190; 
reglamento de la reciente ley nacional pensaron en algún momento adjuntar, como ya se refirió, el texto de ambos protocolos, pero generaron un deplorable vacío normativo al aprobar y publicar solo uno, el de la muerte encefálica.

Artículo 6․- Muerte Accidental

En caso de muerte accidental donde por ley se deba practicar la necropsia y previo al levantamiento del cadáver, es permisible la ablación de órganos o tejidos para fines de trasplante, siempre y cuando no obstaculice el resultado de la investigación de ley. El informe de los hallazgos operatorios será incluido en el Certificado de Necropsia.

Este preciso artículo nos exime de cualquier comentario.

Artículo $7^{\circ}$.- Protocolo de diagnóstico de muerte encefálica

El diagnóstico de muerte encefálica se efectuará de acuerdo al protocolo siguiente:

a) Determinación de la causa básica.

b) Coma arreactivo estructural e irreversible, con asistencia respiratoria mecánica y estabilidad hemodinámica ya sea espontánea o con ayuda de drogas vasoactivas, u otras sustancias, descartando la presencia de hipotermia, sustancias depresoras del sistema nervioso central, o paralizantes que puedan ser causantes de coma o contribuir al cuadro clínico.

El protocolo de diagnóstico de muerte encefálica en el Perú comprende nueve ítems, cada uno de ellos identificado con letras que van de la $a$ hasta la $g$. Antes de comentar los dos primeros, me parece oportuno mencionar que desde que se definió el concepto de muerte cerebral, en la década del 60 del siglo pasado, fue evidente que los criterios legales para certificarla tenían algunas diferencias de un país a otro, aunque tal desigualdad no era significativa en los esenciales criterios generales mostrados en el recuadro, sino en algunos procedimientos complementarios del diagnóstico.

Tal característica se mantiene hasta la actualidad, no obstante que con el tiempo, tanto en el Perú como en otros lugares, los criterios para certificar la muerte cerebral han incorporado razonables modificaciones. Así, en una publicación del año 2002 se reportaron algunos datos interesantes obtenidos al revisar el juicio diagnóstico de muerte cerebral, correspondiente a ochenta países. ${ }^{20}$ Se encontró que 55 de las ochenta naciones —es decir, un 69\%contaban con leyes específicas para normar el trasplante de órganos cadavéricos. Y en setenta de ellas (88\%), el diagnóstico de muerte cerebral se sustentaba en

Machado C y la Comisión nacional para la determinación y certificación de la muerte en Cuba. Rey Neuro12003; 36: 763-770.

20 Wijdicks Eelco F M. Brain death worldwide. Accepted but no global consensus in diagnostic criteria. Neurology 2002; 58: 20-25. 
lo establecido por ley. Por otro lado, se descubrió que no existía el recomendable consenso global entre los criterios de diagnóstico aceptados en cada lugar, siendo destacable tal desigualdad, básicamente, en algunos de los procedimientos para diagnosticar la muerte cerebral, tales como el tiempo de espera en las reevaluaciones, el requerimiento del test de apnea y el uso de pruebas auxiliares confirmatorias del diagnóstico. El autor de la citada inspección opinó justificadamente que debía considerarse la opción de adoptar criterios estándar en el ámbito internacional, para diagnosticar la muerte encefálica.

Las dos primeras letras del artículo 7 de la ley que estamos examinando destacan dos acreditadas ideas en el tema. La primera es la exigencia de precisar la causa básica que precedió a la aparición de la muerte cerebral y además la obligación médica de comprobar que el resultante daño cerebral en tales casos ocasionó lesiones de carácter irreversible a la estructura del cerebro. La segunda noción está dirigida al cuidadoso deslinde diagnóstico de ciertas afecciones, que pueden ser clínicamente confundidas con la muerte encefálica.

En cuanto a la redacción, opino que debió haberse evitado el uso de la inapropiada expresión coma arreactivo —es cierto que el término no es privativo de la ley peruana-. El coma es el nivel más grave de pérdida de conciencia que atendemos los médicos con relativa frecuencia. No obstante la peligrosidad del trastorno, muchas veces puede ser revertido y, a veces, el paciente se recupera totalmente. La persona en coma, lo mismo que quien está en la condición de muerte encefálica, no tiene perceptividad ni reactividad consciente, pero el coma se identifica fácilmente porque el paciente tiene preservados uno o varios reflejos del tronco encefálico, además de otros reflejos anatómicamente relacionados con otras zonas del sistema nervioso, en tanto que en la muerte encefálica desaparecen todos los reflejos, con excepción de algunos de la médula espinal, que ocasionalmente pueden encontrarse. Por consiguiente, la palabra coma, clínicamente inequívoca, no debe mezclarse con los términos usados para certificar una situación cadavérica (muerte encefálica).

c) Ausencia de reflejos en el tronco encefálico.

1) Pupilas midriáticas o en posición intermedia, sin respuesta a estimulación fótica intensa.

2) Reflejo óculocefálico (no realizar si hay sospecha de fractura cervical).

3) Reflejo óculo-vestibular (no realizar en presencia de otorragia u otorraquia).

4) Reflejo nauseoso.

5) Reflejo tusígeno.

6) Reflejo corneal.

La ausencia de los seis reflejos anotados en el reglamento de la ley es una precisa guía para orientar la investigación clínica de los médicos certificadores hacia la seguridad clínica del diagnóstico de muerte encefálica. Se exploran los 
reflejos enumerados mediante un sencillo examen neurológico. Los detalles técnicos para evaluar los reflejos son aprendidos por los médicos durante su larga formación profesional, y por tal motivo, es explicable que no se describa en la ley el procedimiento de la correcta evaluación de cada uno de ellos.

d) Ausencia de respiración espontánea.

e) Prueba de apnea.

f) Prueba de atropina.

No es posible adivinar lo que pensaron los autores de la ley cuando separaron a la ausencia de respiración espontánea de la prueba de apnea, como si se tratara de dos criterios independientes que contribuyen al diagnostico de la muerte encefálica, cuando es bien sabido que la prueba de apnea es justamente el procedimiento utilizado para comprobar la falta de respiración espontánea. Por consiguiente, los ítems de las letras $d$ y $e$ debieron tener una redacción inteligible, fusionándose en uno solo, que indicara la necesidad de verificar la ausencia de respiración espontánea, incluyendo para tal fin la prueba de apnea. Luego, la letra f dispone la ejecución de la prueba de atropina, cuyo objetivo es comprobar la ausencia, sin retorno, de la respuesta cardiaca, que se explica en los casos de muerte encefálica por la extensión de la necrosis a todo el tronco encefálico, incluyendo la destrucción de los núcleos vagales, cardíacos y vasomotores, ubicados en el bulbo raquídeo, del mismo modo que la ausencia irreversible de respiración es la expresión del daño deletéreo de los centros respiratorios, también localizados en el tronco encefálico.

Es evidente, entonces, que las dos pruebas referidas son para determinar clínicamente la lesión total y definitiva del tronco encefálico y tienen igual significado que la ausencia de los reflejos de la misma estructura destacados con la letra $c$, aunque frecuentemente, como también ha ocurrido en nuestra nueva ley, se acostumbra examinarlos por separado.

g) Opcional al diagnóstico clínico de muerte encefálica, es permisible los estudios de flujo sanguíneo cerebral, en aquellos centros que cuenten con dichos procedimientos.

El contenido de este acápite g es incomprensible. Supongo que esto se debe a la ausencia de varias líneas, no incluidas, del texto original, ya que no es posible aceptar la redacción en los términos mostrados. De acuerdo a ella el diagnóstico clínico de muerte encefálica es opcional, entendiéndose que es permisible prescindir de él, cuando se pueden realizar estudios de flujo sanguíneo cerebral (¿?). Revisando la legislación extranjera sobre donación de órganos y tejidos, encontramos que en algunos países, como Cuba y España, se han adoptado recientes modificaciones en la certificación de la muerte encefálica, cuyo objetivo es acortar, cuando es factible, el tiempo del período de observación, con el 
fin de optimizar los resultados del trasplante de órganos cadavéricos provenientes de sujetos en muerte encefálica. Pero desde luego en ningún país se acepta considerar como opcional el esencial diagnóstico inicial de muerte encefálica, con el cual se inicia el período de observación. Citemos como ejemplo la normatividad española de $1999^{21}$ y la cubana de $2001,{ }^{22}$ debido a que la nueva ley peruana tiene notorias coincidencias con ellas. En el artículo 10 del real decreto ibérico se lee lo siguiente:

El cese irreversible de las funciones encefálicas esto es, la constatación de coma arreactivo de etiología estructural conocida y carácter irreversible se reconocerá mediante un examen clínico adecuado tras un período apropiado de observación. Los criterios diagnósticos clínicos, los períodos de observación, así como las pruebas confirmatorias que se requieran según las circunstancias médicas se ajustarán a los protocolos incluidos en el Anexo I del presente Real Decreto.

En este artículo se puntualiza la obligatoriedad de adecuarse tanto a los criterios de diagnóstico como a los períodos de observación. Hace algunos años, y aún ahora en muchos países, se disponía como criterio de diagnóstico de muerte encefálica, la ejecución de reevaluaciones, es decir que no bastaba con efectuar un diagnóstico clínico inicial sino que se exigía la repetición de un segundo examen, confirmatorio, el cual debía efectuarse no antes de las horas fijadas por las leyes nacionales, particulares. La vigente ley española no utiliza la palabra reevaluación sino períodos de observación, los cuales varían entre seis y 48 horas según la situación, pero la idea es esencialmente la misma: otorgar al diagnóstico de muerte de la persona, mediante la doble comprobación de la muerte encefálica, la absoluta garantía de idoneidad que la sociedad espera.

Así, tanto en la ley española como en la cubana, el protocolo para diagnosticar la muerte encefálica en adultos y niños mayores de dos años —en ambos casos hay una gran analogía con los criterios que venimos comentando de nuestra ley- exige que el período de observación entre la primera y segunda exploración se cumpla no antes de las seis horas. Luego, la ley ibérica dictamina que: «[...] los períodos de observación pueden reducirse si se dispone de una prueba complementaria adicional», lo cual significa que la segunda evaluación clínica puede omitirse y en su reemplazo es legalmente admisible, realizar antes de las seis horas, alguna prueba opcional complementaria, en especial aquellas que

21 Real Decreto 2070/1999, del Ministerio de la Presidencia, por el que se regulan las actividades de obtención y utilización clínica de órganos humanos y la coordinación territorial en materia de donación y trasplante de órganos y tejidos. BOE número 3, enero del 2000; 179-190.

22 Machado C. y la Comisión Nacional para la Determinación y Certificación de la Muerte en Cuba. Rey Neuro12003; 36: 763-770. 
miden el flujo sanguíneo cerebral. En definitiva, para establecer la muerte encefálica tienen igual valor, ya sea las dos confirmaciones clínicas del diagnóstico, con seis horas de separación entre ellas, o solo la inicial evaluación clínica y en vez de la segunda investigación, una prueba auxiliar confirmatoria, antes de las seis horas de observación.

En mi opinión, esta parte de la ley española — de modo similar a la cubanaaprobando legalmente el recorte del período de observación, al colocar en la condición de opcional a la anteriormente confirmatoria segunda evaluación clínica, aunque esté motivada por la atendible necesidad de realizar trasplantes precoces en espera de mejores resultados para la supervivencia del paciente receptor, es una cuestionable disposición en la normatividad sobre el tema, y por tal razón, es posible que su aceptación no se extienda fácilmente a otros países. Desde luego que valorando el punto $g$ del protocolo de la ley peruana, la calificación es deplorable, ya que de acuerdo a lo escrito, se ha hecho caso omiso a la, internacionalmente aconsejable, norma que obliga legalmente a las reevaluaciones o períodos de observación. No menos criticable es que el fundamental diagnóstico clínico inicial de muerte encefálica esté considerado un criterio opcional, y en consecuencia, pueda ser reemplazado por estudios del flujo sanguíneo cerebral, con lo cual se deduce que la muerte encefálica puede ser certificada sin necesidad del examen clínico del paciente (¿?). Reitero las palabras iniciales de esta sección, en el sentido de que bien puede tratarse de un desacierto generado al transcribir el texto en referencia y lo que quisieron decir los autores, es que (solo) la segunda evaluación clínica, igual que en España y Cuba, puede convertirse en opcional.

Artículo $8^{\circ}$.- Protocolo de diagnóstico de muerte encefálica en caso de niños Para efecto del diagnóstico de muerte encefálica en caso de niños, adicionalmente a los criterios señalados en el artículo precedente, es indispensable:

a) Hacer el diagnóstico diferencial con: trastornos metabólicos, intoxicaciones, síndrome Guillain Barré hiperagudo, botulismo, síndrome de casi ahogamiento, hipotermia.

b) Se realizará un período de observación en función a la edad:

- Recién nacidos con más de 38 semanas: una semana después de la injuria

- 7 días a dos meses: 2 evaluaciones clínicas con intervalos de 48 horas

- 2 meses a un año: 2 evaluaciones clínicas entre 24 horas

- Mayor de un año: observación 12 horas

- En encefalopatías hipóxico isquémicas se recomienda 24 horas de observación

Después de muchos años, la legislación peruana ha admitido, con censurable retraso, el indispensable ordenamiento de los asuntos relacionados con el diagnóstico de muerte encefálica en niños. Sin embargo, notamos que en el encabezamiento del artículo 8 no se puntualiza, como debe esperarse, la edad 
máxima de la niñez abarcada por la ley. En España y Cuba, los criterios de diagnóstico de muerte encefálica aplicables a los niños mayores de dos años son iguales a los aceptados para los adultos, de modo que para los fines de la ley sobre donación y trasplantes, se exigen particulares requisitos únicamente a los niños menores de dos años. Es posible que no obstante la mencionada omisión de la edad en la ley peruana, el pensamiento de los autores de la misma haya sido similar al de sus pares foráneos, ya que en la secuencia de la sección $b$ del artículo en revisión, están señalados detalladamente los criterios de diagnóstico de muerte encefálica, aplicables particularmente a niños menores de dos años. La excepción a esta conjetura es la penúltima frase, referida simplemente al niño mayor de un año, pero sin fijar como corresponde el tope máximo de la edad infantil comprendido en la disposición.

Empieza el artículo 8 recomendando que antes de etiquetar el diagnóstico de muerte encefálica, debe descartarse la presencia de conocidas enfermedades que pueden llegar a confundirse con tal estado, agregando el nombre de tales patologías. Luego se dispone la obligatoria realización de períodos de observación antes de aceptar el diagnóstico definitivo de muerte encefálica — recordemos la ausencia de esta aconsejable norma en el protocolo nacional de muerte encefálica para adultos, discutido anteriormente-.

El tiempo correspondiente al período de observación varía en función de la edad, de acuerdo a las siguientes pautas:

- Recién nacidos con más de 38 semanas. La redacción de esta parte del reglamento de ley es incorrecta e incomprensible. Dice escuetamente, una semana después de la injuria. Tal frase puede significar que el niño de la edad aludida, en caso de tener una injuria que le provoque en un primer momento el estado clínico compatible con muerte encefálica, debe permanecer en la misma condición, hasta una semana después, cuando se cumpla con la perentoria segunda evaluación, confirmatoria del diagnóstico. Pero además debe cumplirse con la disposición que aparece posteriormente en el reglamento, destacando que para diagnosticar muerte encefálica en los niños menores de un año, es obligatoria la realización de exploraciones complementarias (electroencefalograma, flujo sanguíneo cerebral o gammagrafia de perfusión).

- Siete días a dos meses. En este punto el reglamento aprobado es similar a los criterios aplicables internacionalmente en niños recién nacidos hasta los dos meses de edad. Se demanda, como en todas partes, la obligatoriedad de realizar dos evaluaciones clínicas con un intervalo no menor que 48 horas. En otros lugares se exige, además, la toma simultánea de dos trazados electroencefalográficos para definir el diagnóstico. Pero en el reglamento de la ley nacional, aparentemente esto no es indispensable, ya que solo se 
alude a la realización de alguna de las tres exploraciones complementarias referidas, sin mencionar, inexplicablemente, las insoslayables pautas del caso.

- Dos meses a un año. En estas edades, las normas internacionales aconsejan que el diagnóstico de muerte encefálica conste obligatoriamente de dos evaluaciones clínicas separadas por un tiempo no menor de 24 horas y asimismo de dos electroencefalogramas, aunque la segunda exploración y el segundo electroencefalograma pueden omitirse si se detecta ausencia de flujo sanguíneo cerebral mediante una prueba complementaria adecuada. En la ley peruana se puntualiza igualmente la necesidad de comprobar el diagnóstico de muerte encefálica mediante dos exámenes clínicos separados por 24 horas, pero en este grupo de edades, lo mismo que en el precedente, tampoco se han considerado reglas claras respecto del uso del electroencefalograma como complemento diagnóstico y solo de modo indeterminado se le incluye entre alguna de las tres pruebas complementarias de realización obligatoria en menores de un año.

- Mayor de un año. Este segmento de ley peruana es incoherente. Debió decir de uno a dos años de edad y ser redactado de modo conciso. La vaguedad del texto es notoria al decir sencillamente: observación doce horas. Y agregar en punto aparte: en encefalopatías hipóxico isquémicas se recomienda 24 horas de observación. Tal escrito contrasta con la ejemplar claridad de las normas aprobadas en países con liderazgo internacional en el tema, las cuales estamos seguros fueron revisadas por los autores de nuestra ley. Así, en la normatividad cubana sobre el tema se lee: «Entre uno y dos años. Dos exploraciones clínicas separadas por doce horas —en presencia de lesión destructiva - o 24 horas - cuando la causa del coma es encefalopatía anoxicaisquémica- Estos períodos de observación pueden reducirse si se dispone de una prueba diagnóstica adicional». ${ }^{23}$

c) Existen condiciones que obligan a la realización de exploraciones complementarias: Electroencefalograma, flujo sanguíneo cerebral o gammagrafía de perfusión, para el diagnóstico de muerte cerebral.

- Niños menores de un año

- Ausencia de lesión estructural del encéfalo, demostrable por evidencia clínica o por neuroimagen.

- Lesiones infratentoriales

Esta sección del reglamento tiene también una redacción confusa y ha sido además incluida por error en el artículo 8, que trata estrictamente sobre el protocolo de diagnóstico de muerte encefálica en caso de niños, cuando en realidad se trata de recomendaciones generales, no solo aplicables a ellos. En 
efecto, se sabe que en algunas situaciones patológicas graves pueden suscitarse dudas respecto de la seguridad del diagnóstico clínico de muerte encefálica. Por tal motivo, en la legislación de varios países se ha agregado un acápite individual, fijando las normas aplicables a la pérdida irreversible de las funciones encefálicas en situaciones especiales — niños con menos de un año, ausencia de lesión estructural y lesiones infratentoriales directas-. En estos casos, además del examen neurológico, se exige la ejecución de al menos una prueba complementaria de diagnóstico instrumental.

Artículo $9^{\circ}$.- Acta de comprobación de muerte encefálica

El Acta de Comprobación de muerte encefálica se levantará en el formato que figura como anexo $\mathrm{N}^{\circ} 1$ del presente Reglamento, la misma que será suscrita por los profesionales a que se hace referencia en el artículo $4^{\circ}$ del presente Reglamento, de acuerdo al protocolo establecido en los artículos $7^{\circ}$ y $8^{\circ}$ precedentes.

El anexo 1 de comprobación de muerte encefálica, aunque lleva el disonante encabezado "Acta de muerte cerebral», contiene los datos suficientes para dejar constancia de la muerte de una persona que es encontrada en estado de muerte encefálica. La sociedad debe confiar en que cada uno de los tres médicos firmantes del acta sustentará la seguridad de su certificación, en el celoso conocimiento y cumplimiento de los criterios señalados como requisitos legales para llegar al diagnóstico de muerte encefálica. Esta obligación profesional y moral es, asimismo, el mayor incentivo para leer y releer la ley 28189, con el fin de conocer a cabalidad su contenido, para recomendar, cuando sea pertinente, la corrección de los errores conceptuales y de redacción que puedan detectarse - tal como hemos hecho en este artículo-, y para aportar ideas con el fin de perfeccionar el texto legal, de modo que la claridad de las normas reduzca los posibles conflictos durante su aplicación.

Artículo $10^{\circ}$.- Embalsamamiento o incineración del cadáver

Cuando por disposición de la persona en vida o por voluntad de sus familiares se proceda al embalsamamiento o incineración del cadáver, es permisible la ablación de tejidos no regenerables con fines de trasplante.

Este artículo no será comentado ya que evidentemente no tiene ninguna relación con el título II que está dedicado al diagnóstico de muerte. Me parece que la ubicación del mismo debió ser en el título III de la ley referido a la donación, extracción y trasplante. 